\title{
Industrial Processes Automation in the Pilot Plant at the University of Camagüey
}

\section{Francisco Pacheco Ballagas ${ }^{1}$, Orlando Regalón Anias ${ }^{2}$, Milagros Diez Rodríguez ${ }^{3}$, Vladimir Rodríguez Diez ${ }^{4}$, Raúl Báez Prieto ${ }^{5}$, José Gerardo Baltá García ${ }^{6}$}

1,2,3,4,5 Universidad de Camagüey- Departamento Ing. Eléctrica de la Facultad Electromecánica. Universidad de Camagüey, Cuba.

Email: francisco.pacheco@reduc.edu.cu, orlando.regalon@reduc.edu.cu,milagros.diez@reduc.edu.cu,

vladimir.rodriguez@reduc.edu.cu,raul.bprieto@reduc.edu.cu

Received: November $24^{\text {th }}, 2017$.

Accepted: November $30^{\text {th }}, 2017$

Published: December $13^{\text {th }}, 2017$.

Copyright $@ 2016$ by authors and Institute of Technology Galileo of Amazon (ITEGAM). This work is licensed under the Creative Commons Attribution International License (CC BY 4.0). http://creativecommons.org/licenses/by/4.0/

\begin{abstract}
In this assignment are viewed general aspects of the automation of the malt and beer elaboration process in the pilot plant in the University of Camaguey, for that is made the analysis function of the different devices that take part in obtaining the beverage, explaining, this way, the cycles that this process has. For the plant automation, it was designed and implemented the measurement and actuation of the system, giving the user so much more comprehension of the plant functioning process. The design of the automation was carried out by a PLC MasterK-120S DR20U and for supervision and control it was used an SCADA (Supervisory Control and Data Acquisition), EROS v5.10. As a result, it is obtained a process that accomplishes the expected results, more reliable, safe and efficient.
\end{abstract}

Keywords: Automation, Industrial process, Control.

\section{RESUMEN \\ Automatización de Procesos Industriales en la Planta Piloto de la Universidad de Camagüey}

En este trabajo se abordan los aspectos generales de la automatización de los procesos de elaboración de malta y de cerveza en la planta piloto de la Universidad de Camagüey, para ello se hace un análisis del funcionamiento de los diferentes equipos que intervienen en la obtención de estas bebidas, explicando así las etapas con que cuenta el proceso. Para la automatización de la planta se diseñó e implementó el sistema de medición y actuación, dándole al operario una mayor comprensión del funcionamiento del proceso en la planta. El diseño de la automatización se realizó con ayuda de un PLC MasterK-120S DR20U y para la supervisión y control se utilizo un SCADA (Supervisory Control and Data Adquisition) EROS v5.10.

Palabras Claves: Automatización, Proceso industrial, Control.

\section{INTRODUCCIÓN}

El uso intensivo del control automático de procesos tiene como origen la evolución y tecnificación de las tecnologías de medición y control aplicadas al ambiente industrial. Su estúdio y aplicación ha contribuido al reconocimiento universal de sus ventajas y beneficios asociados al ámbito industrial, que es donde tiene mayor uso en aplicaciones, debido a la necesidad de controlar um gran número de variables, sumado esto a la creciente complejidad de los sistemas.

El control automático de procesos se usa fundamentalmente porque reduce el costo asociado a la generación de bienes y servicios, incrementa la calidad y volúmenes de producción de una planta industrial entre otros beneficios asociados con su aplicación. La eliminación de errores y un aumento en la seguridad de los procesos es otra contribución del uso y aplicación de esta técnica de control. En este punto es importante destacar que anterior a la aplicación masiva de las técnicas de control automático en la industria, era el hombre el que aplicaba sus capacidades de cálculo e incluso su fuerza física para la ejecución del control de un proceso de la máquina asociada a la producción [1].

En nuestro país y en el mundo, el control automático de procesos se utiliza en todas las grandes industria permitiendo aumentar producciones, mejorar la calidad, reducir costos y personal, ejemplo de ello son las cervecerías, en la que se controla 
cada etapa de elaboración, para obtener un produto con la calidad normada [2].

La planta piloto de la Universidad de Camagüey, adjunta a la Facultad de Química cuenta con varias líneas especializadas en la elaboración de productos como encurtidos vegetales, rones, vinos, malta, cerveza, etc. Para producir cerveza y malta se tiene que cumplir la carta de tecnológica (recetas) de estas bebidas, controlando rangos de temperatura en diferentes equipos, lo que se hace manualmente abriendo y cerrando válvulas, lo que resulta extremadamente laborioso, a la vez que en ocasiones la calidad del producto terminado no complace las expectativas por lo que constituye un problema.

Por lo que se propuso diseñar un sistema que permita automatizar el proceso de elaboración de cerveza y malta en la planta piloto porque si se realiza un estudio que permita realizar el proceso de elaboración de cerveza de manera automatizada podríamos incrementar la eficiencia y la calidad del producto.

\section{MATERIALES Y MÉTODOS}

\section{II.1CARACTERÍSTICAS DEL PROCESO DE LA PRODUCCIÓN DE MALTA}

El proceso de producción de la malta consta de 2 etapas fundamentales:

- Etapa en caliente;

- Etapa en frio.

En la etapa en caliente se realizan los procesos de limpieza y molienda de la cebada malteada, maceración, extracción del mosto, cocción del mosto, sedimentación. En la etapa en frío se realiza en dos operaciones, enfriamiento de la malta en el sedimentador, enfriamiento em el reactor y el gaseado $o$ carbonatado de la malta [3].

\section{II.2 PROCESO DE PRODUCCIÓN DE MALTA EN LA PLANTA PILOTO}

La planta piloto cuenta con los siguientes procesos para la fabricación de malta, estos procesos son manuales. Guiándonos por el diagrama de flujo los procesos son.

Molienda (manual)

- Maceración (manual);

- Filtrado (manual);

- Cocción del mosto(manual);

- Sedimentación (manual);

- Enfriamiento (manual);

- Gaseado(manual).

Actualmente, el control de la producción de malta en cada uno de estos procesos se realiza en gran parte de forma manual. Se cuenta con un panel principal desde donde, por medio de botoneras de arranque y parada se comanda el encendido o el apagado de cada uno de los equipos involucrados en el proceso. Las secuencias para encender o apagar los motores de los equipos en los diferentes processos dependen estrictamente del criterio de la persona que opera el proceso.

\section{II.3 PASOS PARA LA PRODUCCIÓN DE LA MALTA}

Veinticuatro horas antes de la arrancada de la planta para la producción de malta es necesario poner en funcionamiento el sistema de refrigeración. En el banco de hielo se enfría el agua alcoholada a uma temperatura de $-8^{\circ} \mathrm{C}$ para que esté en condiciones para poder bajar la temperatura de la malta en el reactor hasta (-1 $2)^{\circ} \mathrm{C}$ y se sensa la temperatura con un PT-100 y si alcanza uma temperatura de $-10^{\circ} \mathrm{C}$ se apaga la compresor de refrigeración y la arranca cuando alcanza una temperatura de $-5^{\circ} \mathrm{C}$. El control de la temperatura del agua alcoholada que se realiza manualmente es conocido como "on-off".

En el macerador con capacidad de 150 litros se el agua de proceso. La cebada molida se vierte en el macerador donde se controlan las temperaturas y las pausas siguiendo la carta tecnológica. La fuente de energía que garantiza la temperatura necesaria para el proceso de maceración proviene de la caldera de la Universidad de Camagüey, este vapor es regulado en um lazo compuesto por una válvula auto operada donde se regula la presión hasta $1.5 \mathrm{~kg} / \mathrm{cm} 2$. El vapor de agua circula por la chaqueta del macerador; y la presión de vapor de agua se sensa a través de un manómetro. La temperatura se sensa com un PT-100. El macerador cuenta con un agitador de paleta que está acoplado a un motor a través de un reductor por transmisión de correa y polea, este hace mover el agitador com el objetivo de que la temperatura del caldo de la malta sea homogénea. Al haber terminado el proceso de maceración se abre la válvula que está acoplada a la salida del macerador para vaciar el caldo de la malta hacia un colector de mosto de 150 litros donde se filtra mientras que se va vaciando. Después de haber filtrado todo el mosto, se bombea de nuevo hasta el tacho.

Cuando el tacho ya contenga el caldo de la malta se pasa al proceso de cocción y abre la válvula que regula el flujo de vapor para que el caldo alcance una temperatura de $100^{\circ} \mathrm{C}$ em 15 minutos y después mantener $100^{\circ} \mathrm{C}$ durante una hora. Después de la cocción se le acopla la electrobomba sanitaria al colector de mosto para bombearlo hasta el sedimentador de 150 litros, este caldo que ya se le puede llamar malta, y entra de forma tangencial al sedimentador. Después de estar lleno, se arranca la electrobomba que circula agua a temperatura ambiente por la chaqueta del sedimentador hasta que la temperatura de la malta descienda hasta $35^{\circ} \mathrm{C}$ o $40^{\circ} \mathrm{C}$. En esta etapa deben ser controladas tres temperaturas la de la malta contenida en el sedimentador, la de la entrada de agua y la de salida de agua, se utilizan tres PT 100, una para cada lectura.

Luego de esto se abre la válvula lateral para descargar el mosto clarificado hasta el colector de mosto donde se acopla la electrobomba sanitaria para bombear la malta hasta el reactor. Cuando esté lleno el reactor se le hace circular agua alcoholada a una temperatura $(-5 \div-10){ }^{\circ} \mathrm{C}$, mediante el banco de hielo que para este momento deberá tener el agua alcoholada a una temperatura $10^{\circ} \mathrm{C}$ para así lograr que la malta alcance una temperatura de $(1 \div 2)$ ${ }^{\circ} \mathrm{C}$.

Después de haber alcanzado la malta una temperatura de $(1 \div 2){ }^{\circ} \mathrm{C}$ se procede al gaseado de la misma [3][4].

\section{II.4 CARACTERÍSTICAS DE LA ELABORACIÓN DE CERVEZA Y MALTA EN LA PLANTA PILOTO}

Comparación y similitudes:

Para la elaboración de ambas bebidas se utiliza el mismo equipamiento por lo que se utilizan los mismos sensores y actuadores. Las principales diferencias están en el proceso de maceración y la etapa en frio. La maceración no se realiza de igual forma para ambos productos porque estos tienen diferentes cartas tecnológicas. En la etapa en frio la malta consta con dos procesos, enfriamiento y gaseado; la cerveza con tres, fermentación, maduración y enfriamiento. 


\section{II.5 LA AUTOMATIZACIÓN EN LA PLANTA PILOTO}

Para lograr un mejor control de los procesos que intervienen en la elaboración de la cerveza y la malta, en el mundo, se (Controlador Lógico Programable) que mediante un sistema SCADA (Supervision Control and Data Adquisition) possibilita la lectura, procesamiento y almacenamiento de información proveniente de los sensores y a partir de estos se toman decisiones sobre los actuadores en tiempo real [5].

\section{II.5.1 PROCESOS DE MACERACIÓN Y COCCIÓN MACERACIÓN Y COCCIÓN}

Parámetros a tener en cuenta en estos procesos:

1. Temperatura del mosto para poder regular la apertura de la válvula neumática con um conversor electro neumático[6-8].

2. Controlar el motor y su acoplamiento con el agitador.

Dispositivos utilizados para lograr la automatización de estos procesos:

- Un sensor de temperatura;

- Un motor;

- Un contactor;

- Un breaker;

- Tres válvulas manuales;

- Válvula neumática;

- Conversor electroneumático;

- Un transductor con una salida de 4-20 mA [6][9][10];

- Un relé de control;

- Un compressor;

- Un sensor que indique que el agitador está trabajando.

\section{II.5.2 PROCESO DE SEDIMENTACIÓN}

Parámetros a tener en cuenta en este proceso:

1 Temperatura del mosto para poder controlar desde el PLC la electrobomba que circula el agua a temperatura ambiente por la chaqueta del sedimentador.

2 Temperatura de entrada y salida del agua de la chaqueta, para poder hallar la transferencia de calor del mosto al agua.

Dispositivos utilizados para lograr la automatización de estos procesos:

- $\quad$ Tres sensores de temperatura PT100 [11][12];

- Tres transductores para los sensores de temperatura;

- Una electrobomba;

- Una cuchilla;

- Un relé de control.

\section{RESULTADOS Y DISCUSIÓN}

\section{III.1 EQUIPAMIENTO NECESARIO PARA LLEVAR A CABO LA AUTOMATIZACIÓN.}

Analizando los parámetros a tener en cuenta en cada proceso y los equipos que se controlan en los mismos. Para llevar a cabo la automatización, bajo las condiciones actuales, en la Planta Piloto de la Universidad de Camagüey fue necessário adquirir un autómata con al menos 11 entradas y 4 salidas digitales, 8 entradas y 1 salida analógica, una computadora para la supervisión y control desde el SCADA y los accesorios que permitan su interconexión al proceso.

\section{III.2 DISEÑO DEL SISTEMA DE AUTOMATIZACIÓN}

Para el diseño del sistema se definieron las entradas y salidas, digitales y analógicas, así como los respectivos lazos de control con que cuenta la versión automatizada del proceso.

La automatización de los procesos involucrados en la elaboración de las bebidas requirió del empleo de un PLC MasterK-120S DR20U de la firma LG con tres módulos de expansión, dos Modulos G7F-ADHA y un G7F-AD2A, el cual se encontraba disponible en la empresa de servicios CEDAI. Se solicitó la compra del mismo autorizado por el rector de la Universidad de Camagüey.

Para la supervisión y control de los proceso se diseñó en el SCADA (Supervisory Control and Data Adquisition) EROS v5.10, software que se imparte dentro de la asignatura de Automatización Industrial y Autómatas en cuarto año de la carrera de Ingeniería Eléctrica [13].

Para probar la programación y el SCADA se utilizaron las instalaciones del laboratorio de Accionamiento Eléctrico, arrojando bueno resultados estas pruebas.

Debido al equipamiento con que cuenta la planta piloto el proceso de elaboración de las bebidas no puede ser automatizado en su totalidad, por lo que se automatizaron los procesos de manera independiente:

- Limpieza de la cebada malteada (manual);

- Molienda de la cebada malteada (manual);

- Maceración (automático);

- Extracción del mosto (manual);

- Cocción del mosto (automático);

- Sedimentación del mosto (automático);

- Fermentación (automático);

- Maduración (automático);

- Enfriamiento (automático).

\section{III.3 CARACTERÍSTICAS TÉCNICAS DEL PLC MASTERK 120S DR20U}

- El PLC MasterK-120S DR20U consta con 12 entradas de digitales de P00-P0B, y con 8 salidas de P40-P47.

- Consta con 8 reguladores PID (Proporcional Integral Derivativo) digitales incluidos.

- Un puerto serial RS-232 [14].

- Un puerto RS-485 para la comunicación aplicando el protocolo MODBUS.

- Se le pueden conectar tres módulos de expansiones como máximo [15].

\section{III.4 SELECCIÓN DE SENSORES Y ACTUADORES}

La secuencia medir-decidir-actuar es válida tanto para manejar una sola variable como para una planta completa donde medir una propiedad en el producto terminado puede conllevar acciones sobre determinadas operaciones en la línea del proceso. Ahora bien, no solo es necesario realizar mediciones con el propósito de controlar, también es habitual medir otras variables de manera que se tenga una información completa de lo que está sucediendo y transmitir esta información con el objetivo de representarla o almacenarla para ser utilizada posteriormente [16].

El diseño e implementación de los sistemas de medición y actuación se realizó analizando la cantidad de sensores y actuadores necesarios para automatizar el proceso de elaboración de las bebidas. Todos los actuadores para el grado de automatizaciónón que se quería se encontraban en la planta, al igual que la mayoría de los sensores, un sensor fue diseñado y el resto 
donado por empresas del territorio. Los principales parámetros a controlar en la planta son: temperatura, presión y posición por lo que se utilizan sensores de:

- Presión: El instrumento que se utiliza para medir presión em la planta piloto es manómetro, este sensor solo muestra la información del fenómeno;

- Temperatura: El sensor que se utiliza en la planta piloto no indica físicamente el fenómeno físico leído, envía la señal a um transductor el cual convierte la señal leída de la temperatura men un valor de 4-20 mA para poder conectarlo al autómata.m Este tipo de sensor se le denomina instrumentos ciegos. En los procesos que se requiere sensar la temperatura se utilizaron PT-100cada una con su transductor; el tipo de conexión que se utilizo es de tres hilos [6][10][11];

- Posición: Este sensor fue diseñado para saber si el agitador del macerador está girando.

Ver sensores y parámetros en la Tabla I. Reed switch (interruptor de lengüeta) es un interruptor eléctrico activado por un campo magnético. Cuando los contatos están normalmente abiertos se cierran en la presencia de un campo magnético y cuando están normalmente cerrados se abren en presencia de un campo magnético [17].

Tabla 1: Sensores utilizados por processo.

\begin{tabular}{|c|c|c|c|}
\hline Procesos & $\begin{array}{c}\text { Parámetro a } \\
\text { sensar }\end{array}$ & Sensor & $\begin{array}{l}\text { Rango } \\
\left(\mathrm{C}^{\circ}\right)\end{array}$ \\
\hline \multirow[t]{2}{*}{$\begin{array}{l}\text { Maceración } \\
\text {-Cocción }\end{array}$} & $\begin{array}{l}\text { Temp. } \\
\text { mosto }\end{array}$ & Pt-100 & $0-120$ \\
\hline & $\begin{array}{l}\text { Ubicación de la } \\
\text { correa }\end{array}$ & $\begin{array}{l}\text { interruptor } \\
\text { de lengüeta }\end{array}$ & - \\
\hline \multirow[t]{3}{*}{$\begin{array}{l}\text { Sedimentac } \\
\text { ión }\end{array}$} & $\begin{array}{l}\text { Temp. agua de } \\
\text { entrada }\end{array}$ & Pt-100 & $0-100$ \\
\hline & $\begin{array}{l}\text { Temp agua de } \\
\text { salida }\end{array}$ & Pt-100 & $0-100$ \\
\hline & Temp del mosto & Pt-100 & $0-100$ \\
\hline $\begin{array}{l}\text { Banco de } \\
\text { Hielo }\end{array}$ & $\begin{array}{l}\text { Temp del agua } \\
\text { alcoholada }\end{array}$ & Pt-100 & $-10-100$ \\
\hline \multirow[t]{3}{*}{ Reactor } & $\begin{array}{lr}\text { Temp } & \text { agua } \\
\text { alcoholada } & \text { de } \\
\text { entrada } & \end{array}$ & Pt-100 & $-10-100$ \\
\hline & $\begin{array}{lr}\text { Temp } & \text { agua } \\
\text { alcoholada } & \text { de } \\
\text { salida } & \end{array}$ & Pt-100 & $-10-100$ \\
\hline & $\begin{array}{l}\text { Temp de la } \\
\text { malta }\end{array}$ & Pt-100 & $-10-100$ \\
\hline
\end{tabular}

Fuente: Los Autores, (2017).

\section{III.4.1 ACTUADORES}

Los actuadores presentes en la automatización de los distintos procesos son de 2 tipos: (Ver Tabla II) Presión neumática Fuerza motriz eléctrica (motor eléctrico o solenoide). Dependiendo del origen de la fuerza el actuador se denomina (VILDÓSOLA)

Tabla 2: Características de los principales actuadores utilizados.

\begin{tabular}{|c|c|c|c|c|c|c|}
\hline $\begin{array}{l}\text { Motor } \\
\text { electrobomba }\end{array}$ & $\operatorname{In}(\mathrm{A})$ & $\begin{array}{l}\mathrm{Vn} \\
(\mathrm{V})\end{array}$ & $\begin{array}{l}\mathrm{Pn} \\
(\mathrm{kW})\end{array}$ & $\begin{array}{c}\operatorname{Cos} \\
\phi p\end{array}$ & $\begin{array}{l}\text { Frecu } \\
\text { encia } \\
(\mathrm{Hz})\end{array}$ & $\begin{array}{l}\mathrm{Nn} \\
(\mathrm{rpm})\end{array}$ \\
\hline $\begin{array}{l}\text { Moto- } \\
\text { Agitador }\end{array}$ & 1.2 & $\begin{array}{l}220- \\
3 \phi\end{array}$ & 0.55 & 0.85 & 60 & 3260 \\
\hline $\begin{array}{l}\text { Electrobomba } \\
\text { del } \\
\text { Sedimentador }\end{array}$ & 3.5 & 110 & 0.172 & 0.45 & 60 & 3450 \\
\hline $\begin{array}{l}\text { Electrobomba } \\
\text { del Reactor }\end{array}$ & 24.4 & 127 & 1.1 & 0.64 & 60 & 3530 \\
\hline $\begin{array}{l}\text { Compresor de } \\
\text { refrigeración }\end{array}$ & 10 & $\begin{array}{l}220- \\
3 \phi\end{array}$ & & & 60 & \\
\hline $\begin{array}{l}\text { Motor del } \\
\text { Molino }\end{array}$ & 4.5 & $\begin{array}{l}220- \\
3 \phi\end{array}$ & & & 60 & \\
\hline $\begin{array}{l}\text { Electrobomba } \\
\text { Sanitaria }\end{array}$ & & $\begin{array}{l}220- \\
3 \phi\end{array}$ & & & 60 & \\
\hline
\end{tabular}

Fuente: Los Autores, (2017).

\section{III.4.2 CONTACTOR}

La selección de los contactores se realizó teniendo en cuenta dos aspectos fundamentales: (Ver Tabla III)

- La capacidad interruptora de los contactos de fuerza.

- La tensión del circuito de control en el cual deben operar (tensión de alimentación de la bobina).

Tabla 3: Características de los contactores utilizados.

\begin{tabular}{|c|c|c|c|}
\hline Contactor & $\operatorname{In}(A)$ & $\mathrm{V}(\mathrm{V})$ & $\begin{array}{l}\text { Tensión de la } \\
\text { bobina de } \\
\text { control(V) }\end{array}$ \\
\hline $\begin{array}{l}\text { Contactor del } \\
\text { motorreductor }\end{array}$ & 16 & 380 & 220 \\
\hline $\begin{array}{l}\text { Contactor de } \\
\text { electrobomba }\end{array}$ & 16 & 380 & 220 \\
\hline \multirow{2}{*}{$\begin{array}{l}\text { Contactor del } \\
\text { motorreductor } \\
\text { del agitador }\end{array}$} & 25 & 380 & \multirow[t]{2}{*}{220} \\
\hline & 14 & 500 & \\
\hline \multirow{2}{*}{$\begin{array}{c}\text { Contactor de } \\
\text { electrobomba } \\
\text { del } \\
\text { sedimentador }\end{array}$} & 25 & 380 & \multirow[t]{2}{*}{220} \\
\hline & 14 & 500 & \\
\hline \multirow{2}{*}{$\begin{array}{l}\text { Contactor del } \\
\text { compres or } \\
\text { refrigeración }\end{array}$} & 5.5 & 220 & \multirow[t]{2}{*}{220} \\
\hline & 10 & 380 & \\
\hline $\begin{array}{l}\text { Contactor de } \\
\text { electrobomba } \\
\text { del reactor }\end{array}$ & 16 & 380 & 220 \\
\hline
\end{tabular}

Fuente: Los Autores, (2017).

\section{III.4.3 RELÉ}

La selección de los relés de control se realiza teniendo en cuenta dos aspectos fundamentales:

- El número de contactos de control necesarios.

- La corriente de control que deben de operar (corriente nominal que deben conectar y desconectar).

- La tensión del circuito de control en el cual deben operar (tensión de alimentación de la bobina).

La alimentación de los relés disponible en la planta es de $42 \mathrm{~V}$, por lo que se hacía necesario disminuir la tensión de $110 \mathrm{~V}$ o $220 \mathrm{~V}$ al nivel de alimentación de estos relés, también se encontraban disponibles contactores magnéticos con algunos contactos de fuerzas deteriorados, a los que se decidió darle mantenimiento para sustituirlos por los relés, ya que la alimentación de los contactores se correspondía con el nivel de tensión en la planta.

\section{III.5 ARQUITECTURA DEL SISTEMA DE MEDICIÓN Y ACTUACIÓN}

El sistema de medición para la automatización de los processo para la elaboración de la cerveza y malta consta con 11 entradas digitales y 8 analógicas. Por su parte el sistema de actuación tiene 4 salidas digitales y 1 salidas analógicas. Se puede apreciar claramente en la figura 1 todas las entradas que provienen de sensores o transmisores, son compatibles con los niveles admitidos por este PLC; igual situación se tiene con las salidas. 


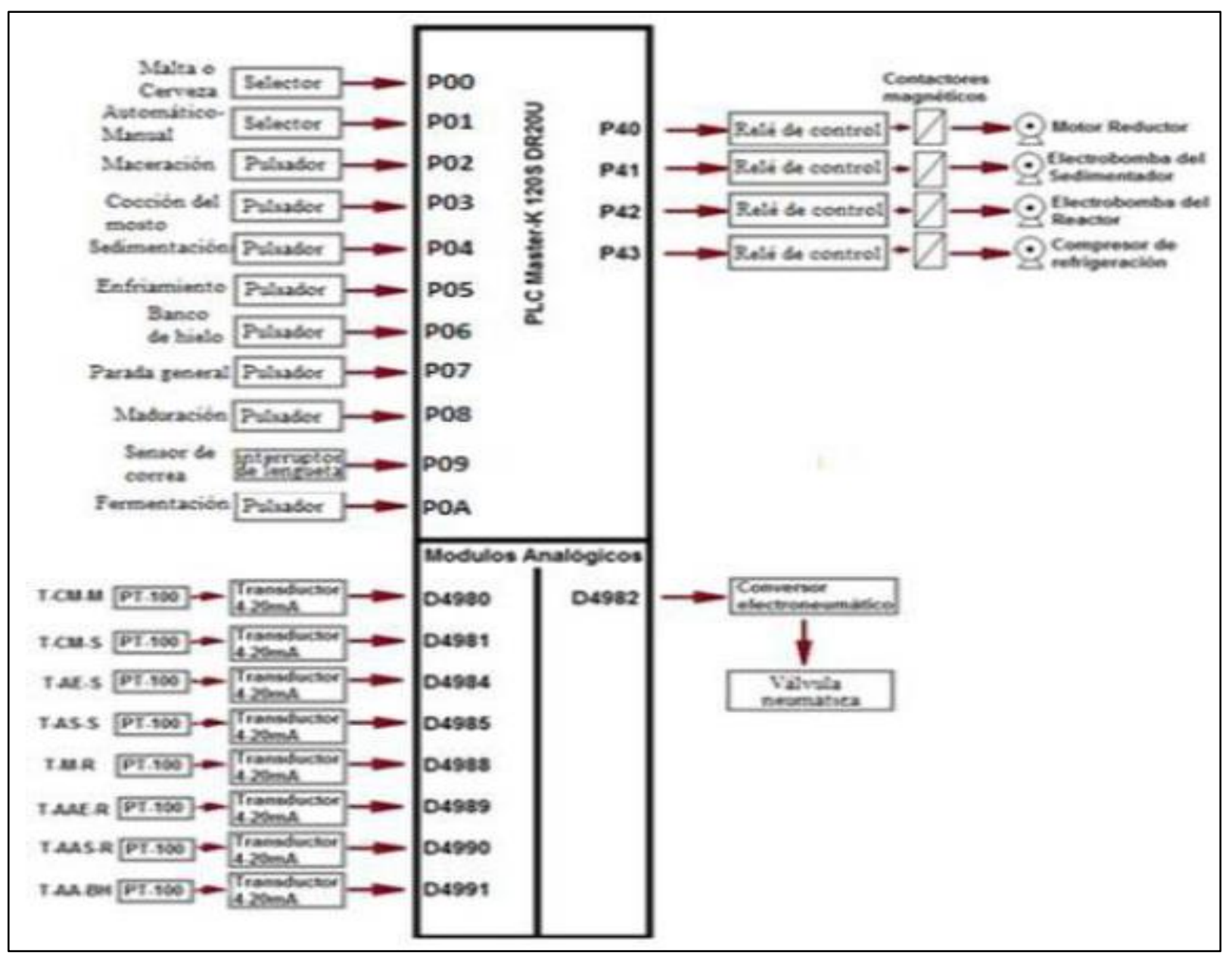

Figura1: Esquema de conexión de las E/S del PLC.

Fuente: Los Autores, (2017).

\section{III.5.1 MODO DE OPERACIÓN}

Como anteriormente se expuso el diseño y montaje del sistema de medición y control se implementó para que funcionara en dos modos: manual y automático.

\section{III.5.2 MANUAL}

Modo en el cuál el operario tiene que conectar las entradas del rastreador de temperatura a los PT-100. Esto permite que el operario pueda ver las temperaturas de los PT-100 instalados a través del rastreador de temperatura y actúe según lo requiera el proceso. En régimen manual también operan desde un panel auxiliar, los procesos de molienda, el compresor y la bomba sanitaria.

\section{III.5.3 AUTOMÁTICO}

Para trabajar en este modo el operario tiene que elegir la opción automática en el panel principal y cambiar el conector de 16 pines del rastreador a los transductores a los PT-100. Este modo le posibilita al operador trabajar desde el SCADA y poder controlar los procesos que se encuentran automatizados. En el panel se encuentran ocho pulsadores y dos selectores.

\section{III.6 LAZOS DE CONTROL}

Uno de los objetivos de la automatización de la planta piloto es controlar la temperatura dentro del macerador, por lo que para el control de temperatura del mosto en los procesos que se realizan en este tacho (maceración y cocción) es necessário implementar dos lazos de control dinámicos, uno para cada proceso, donde intervendrán señales de entrada como de salida. (Ver tabla IV).

Con el fin de determinar las variables que intervienen en el sistema de medición (variables controladas), como en el sistema de actuación (variables manipuladas) se realizó um análisis de cada uno de los lazos de control.

Tabla 4: Caracterización de las variables para cada lazo de control.

\section{Lazo de control de la temperatura del mosto en la maceración:}

\begin{tabular}{ll}
\hline $\begin{array}{l}\text { Variable controlada } \\
\text { (Entrada analógica) }\end{array}$ & $\begin{array}{l}\text { Temperatura del caldo de la } \\
\text { malta }\end{array}$ \\
\hline $\begin{array}{l}\text { Variable manipulada } \\
\text { (salida analógica) }\end{array}$ & Flujo de vapor de agua \\
\hline
\end{tabular}

\begin{tabular}{ll}
\hline $\begin{array}{l}\text { Lazo de control de la temperatura del caldo de la } \\
\text { malta en el proceso de cocción: }\end{array}$ \\
\hline $\begin{array}{l}\text { Variable controlada } \\
\text { (entrada analógica) }\end{array}$ & $\begin{array}{l}\text { Temperatura del caldo de la } \\
\text { malta }\end{array}$ \\
\hline $\begin{array}{l}\text { Variable manipulada } \\
\text { (salida analógica) }\end{array}$ & Flujo de vapor de agua \\
\hline
\end{tabular}

Fuente: Los Autores, (2017). 


\section{CONCLUSIONES}

1 Con el montaje del sistema que permite el control automático-manual de la Planta Piloto se pueden registrar los parámetros fundamentales de temperatura y tiempo y cumplir eficientemente las distintas cartas tecnológicas en para la producción de cervezas y maltas y extensiones.

2 La eficiencia y calidad de las producciones aumentaron exponencialmente, producto del nivel de automatización al que se llegó, demostrando así las ventajas que supone la automatización de cualquier proceso industrial.

3 Con el aumento de la eficiencia y la calidad de los productos ahí producidos la universidad podrá, reducir costos en materia de bebidas y refrescos, para actividades festivas.

\section{REFERENCIAS}

[1] Ogata, Katsuhiko. Ingeniería de Control Moderna. México: Pearson Educación, 2000. 0-13-034281-5.

[2] Guerrero, Eduardo. Procesos Industrial de la Cerveza. Proceso industrial de la elaboración de la cerveza. [Em línea] 4 de septiembre de 2011. [Citado el: 25 de enero de 2014.] http://eduardogr.blogspot.com/2011/09/procesosintermitentescont inuosyotros_04.html.

[3] Benjamin, Yosbel Félix. Evaluación técnico económica de la producción de maltina en la Planta Piloto de la Universidad de Camagüey. Trabajo de pregrado. Camagüey: s.n., 2012.

[4] Ing. Baltá García, José Gerardo. Descripción del Proceso de producción de cerveza y malta de la Planta Piloto. [Mp3] Camaguey: s.n., 2014.

[5] Penin, Aquilino Rodríguez. Sistemas Scada. Marcombo, 2011.

[6] Sole, Antonio Creus. Instrumentación industrial. Marcombo, 2012.

[7] Amaya, Rodolfo Atehortúa. Laboratorio para control automático de proceso. Revista CINTEX, 2014, vol. 4.

[8] García Armada, Elena, et al. Banco de ensayos universal para la evaluación de actuadores. 2012.

[9] Harper, Gilberto Enríquez. El ABC de la instrumentación en el control de procesos industriales. Editorial Limusa, 2000.

[10]Castañeda Verano, Adriana Marcela; Cardona Barón, Carolina María. Convertidor 4-20mA A Profibus-PA. 2010.

[11] Padrón, Eliezer-Fernández; García, Sergio- Fernández; Barrios, Rómulo-Pérez. Aplicaciones industriales. Ingeniería Energética, 2012, vol. 33, no 1, p. 46-58.

[12] González Casallas, Orlando Federico; Moreno Velasco, Víctor Javier. Propuesta de un modelo estadístico paramétrico para la validación de termómetros de resistencia PT100. 2014.

[13] Boyer, Stuart A. Scada: supervisory control and data acquisition. International Society of Automation, 2009.
[14] Rani, Sarita; Malhotra, Smridhi; Sangwan, Vinesh. Detailed Study of RS-232 Serial Interface. International Journal of Research, 2014, vol. 1, no 9, p. 1187-1192.

[15] LG Industrial Systems. LG Programmable Logic Controller MASTER-K. Instructions \& Programming. 2005.

[16] M, Chilikin. Accionamientos Eléctricos. Moscú: Mir Moscú, 1972. 129820

[17] ELECTRONICS MECCANO. Reed Switches. [En línea] 6 de junio de 2000. [Citado el: 18 de junio de 2014.] http://www.eleinmec.com/article.asp?23.

\section{APENDICE}

ANEXO A

ESQUEMA DE CONEXIÓN REALIZADO PARA LA COMUNICACIÓN Y PROGRAMACIÓN

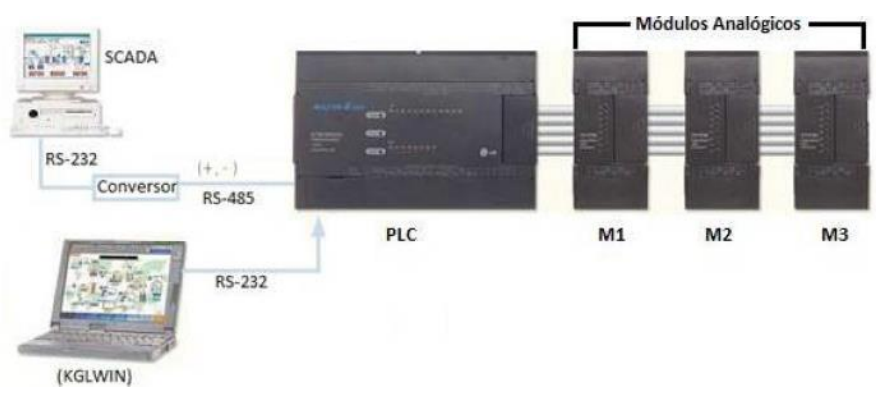

Módulos analógicos:

M1-M2 Son módulos mixtos, de dos entradas y uma salida que pueden ser en forma de voltaje o corriente. Tipo G7F-ADHA.

M3-Este es un módulo tipo G7F-AD2A de cuatro entradas analógica, que pueden ser de voltaje o corriente. 